\title{
e-Phaïstos
}

e-Phaïstos

Revue d'histoire des techniques / Journal of the history

of technology

IV-1 | 2015

Les arts de guerre et de grâce (XIVe-XVIIle siècles)

\section{La joute, le plus gracieux des arts de la guerre}

Joust, most gracious of war art

\section{Loïs Forster}

\section{(2) OpenEdition}

Journals

Édition électronique

URL : http://journals.openedition.org/ephaistos/878

DOI : 10.4000/ephaistos.878

ISSN : 2552-0741

Éditeur

IHMC - Institut d'histoire moderne et contemporaine (UMR 8066)

Édition imprimée

Date de publication : 1 avril 2015

ISSN : 2262-7340

\section{Référence électronique}

Loïs Forster, « La joute, le plus gracieux des arts de la guerre », e-Phaïstos [En ligne], IV-1 | 2015, mis en ligne le 01 avril 2015, consulté le 19 avril 2019. URL : http://journals.openedition.org/ephaistos/878 ; DOI : 10.4000/ephaistos.878

Ce document a été généré automatiquement le 19 avril 2019

Tous droits réservés 


\section{La joute, le plus gracieux des arts de la guerre}

Joust, most gracious of war art

\section{Loïs Forster}

\section{NOTE DE L'ÉDITEUR}

Les résumés en français et en anglais sont rédigés par la rédaction.

1 Par son ambivalence entre martialité et réjouissance nobiliaire, la joute s'avère être un cas intéressant dans l'alternance des arts abordés dans ce colloque; la conjonction de savoir-faire militaire et de mise en scène parfois savante m'a conduit à la désigner, pour l'occasion de ce colloque, comme le plus gracieux des arts de la guerre. Elle constitue effectivement la pratique martiale la plus codifiée de la fin du Moyen Âge, une pratique d'autant plus intéressante et remarquable qu'elle adapte à un contexte festif et courtois gracieux dirons-nous - la technique d'escrime à la lance caractéristique de la chevalerie.

2 La joute résulte ainsi d'une tension entre le spectacle et l'entraînement guerrier, un mélange heureux qui lui a valu au Moyen Âge et lui vaut encore aujourd'hui une immense popularité. Quelle incroyable puissance évocatrice que celle de l'image du chevalier en armure, monté sur son fougueux destrier, en train de courir des lances pour l'honneur des dames! Il suffira pour s'en convaincre de songer à la fréquence de la figure du chevalier chargeant à la lance dans les romans arthuriens, dans les textes comme dans les enluminures des manuscrits. Le pouvoir de fascination de cette activité, actif sur les hommes $\mathrm{du} \mathrm{XV}^{\mathrm{e}}$ siècle, s'applique également aux spectateurs actuels. Les sensations fortes qu'elle véhicule trouvent un écho qui est toujours d'actualité : Brian Helgeland a ainsi introduit les courses des jouteurs par l'air entraînant de We will rock you de Queen, dans son film tout entier consacré à l'univers des joutes, A knight's tale (titre français : Chevalier, 2001). Occupant une place centrale dans l'imaginaire collectif, le "grand tournoi » constitue souvent un temps fort des fêtes médiévales devenues désormais 
populaires dans les villes françaises ${ }^{1}$. Il s'agit généralement de spectacles scénarisés, assurés par des cascadeurs qui ne sont pas des amateurs passionnés mais des professionnels, avec une exigence de rentabilité et une priorité donnée au plaisir du public bien avant le plaisir personnel ou la rigueur historique. Or il existe également une autre forme de joute, à laquelle nous nous intéresserons ici davantage et que nous pourrons qualifier de compétitive, dont les pratiquants cherchent le plaisir personnel, la performance ou la victoire plus que le plaisir du public: pour ceux-ci, la joute est actuellement en train de redevenir, ou de devenir (toute la question est là), un sport. Cette deuxième forme de pratique nous offre un point de comparaison plus intéressant que la première avec le milieu de la reconstitution historique, dont un article d'olivier Renaudeau a montré l'évolution, de l'évocation franchement approximative des années 1980 à la stricte rigueur de certaines associations des années $2000^{2}$. Le domaine des joutes accuse un retard important dans cette évolution et, plus encore, rien n'indique que le chemin suivi soit le même. Ce monde des jouteurs demeure relativement à part et se mélange peu avec le milieu des "reconstituteurs", leurs objectifs étant sensiblement différents.

3 Face à cette tendance à une certaine segmentation entre reconstituteurs, jouteurs sportifs, pratiquants d'Arts Martiaux Historiques Européens ${ }^{3}$, il convient de relever que l'étude de l'art de la joute demande des capacités, ou du moins une compréhension, dans plusieurs domaines : le maniement des armes, le port de l'armure, l'équitation. Or il serait absurde de penser que ces domaines peuvent être abordés d'une façon purement empirique, en cherchant l'efficacité dans une activité reconstituée avec un grand nombre de concessions et de limitations, sans effectuer un travail rigoureux de recherche historique.

4 Afin de saisir l'apport et les perspectives d'une pratique expérimentale de la joute, nous nous intéresserons aux conditions dans lesquelles se pratique la joute, puis à la maitrise technique qu'elle requiert, et enfin à l'état d'esprit dans lequel les jouteurs s'adonnent à cette activité. Pour chacune de ces parties, nous analyserons d'une part la joute médiévale et d'autre part les pratiques actuelles qui visent à la reconstituer (ou du moins à l'évoquer).

\section{Les conditions techniques de la joute}

Même si le sujet du présent article n'est pas de détailler l'évolution des tournois et des joutes, largement étudiés par ailleurs, il convient ici d'en dépeindre rapidement le contexte d'apparition et de brosser à grands traits leur histoire. Pour les chevaliers, dont la place dans la société médiévale est directement liée au savoir-faire militaire, l'entraînement lors de jeux martiaux s'impose comme une nécessité - même si ceux-ci s'avèrent être aussi une distraction fort appréciée et éventuellement une façon de faire fortune et de favoriser sa carrière. Cet entraînement se fait dès le $\mathrm{XI}^{\mathrm{e}}$ siècle lors de tournois très proches de la guerre: les armes utilisées (épées et lances principalement) sont les mêmes, l'affrontement se fait de façon collective, les équipes ne sont pas strictement équilibrées - loin s'en faut parfois - et le terrain n'est absolument pas délimité. Au final, seul l'objectif des participants diffère de la guerre, puisqu'il y est question de faire des prisonniers mais en aucun cas de tuer son adversaire ${ }^{4}$.

6 A partir du XIII ${ }^{e}$ siècle, le tournoi cède progressivement la place à la joute, un affrontement individuel de deux chevaliers à cheval armés d'une lance. Au fil des siècles, 
la place accordée au décorum s'amplifie jusqu'à dénaturer la joute, qui se trouve progressivement vidée de sa substance aux XVI ${ }^{\mathrm{e}}$ et XVII ${ }^{\mathrm{e}}$ siècles, tandis qu'à la guerre les charges de cavalerie lourde sombrent dans l'obsolescence face à l'efficacité croissante des armes à feu et des grandes formations d'infanterie ${ }^{5}$.

Pendant la période faste des joutes, que l'on peut considérer du XIII ${ }^{\mathrm{e}}$ au $\mathrm{XV}^{\mathrm{e}}$ siècle ${ }^{6}$, le lien avec la guerre reste important : la joute transpose dans un cadre normé les charges à la lance pratiquées sur les champs de bataille en groupe serré. Le faible nombre d'adversaires dans la joute rend l'action facile à suivre et suscite l'enthousiasme du public. De plus en plus, les lices peuvent être complètement fermées. Le terrain nécessaire, relativement réduit, permet à ces rencontres de se tenir facilement dans des villes, à moins que la volonté de disposer d'un espace plus étendu pour la réception des concurrents et le déroulement des combats ne conduisent les organisateurs à opter pour la campagne.

8 Les joutes se pratiquent d'abord en champ « ouvert ", c'est-à-dire que les jouteurs peuvent circuler librement à l'intérieur de l'enclos destiné aux combats. A la fin du Moyen Âge, l'usage de la "toile» se répand ${ }^{7}$; il s'agit d'une barrière centrale dont l'invention semble d'origine portugaise ou espagnole, parfois recouverte d'un tissu qui lui donne son nom ${ }^{8}$. L'intérêt majeur de cette séparation est d'empêcher la collision frontale entre les deux chevaux durant leur course, accident redouté en raison des graves séquelles potentielles pour les animaux ${ }^{9}$. Faire accepter à sa monture de croiser au plus près un autre cheval au galop est un travail de dressage assurément difficile, qui constitue pourtant un facteur majeur de la réussite d'une course ${ }^{10}$. Or les chevaux suffisamment habitués et mis en confiance pour s'en remettre totalement aux ordres de leurs cavaliers en oubliant leur peur instinctive, peuvent en venir à se percuter. Notre société contemporaine acceptant plus facilement une prise de risque volontaire que les blessures infligées aux animaux, les joutes actuellement tenues dans une forme compétitive adoptent toutes cet équipement de sécurité important.

9 La sécurité est effectivement un élément à ne pas prendre à la légère dans une activité telle que la joute. Voici ce qu'en dit Jean de Bueil dans les années 1460 à travers la bouche du héros de son roman Le Jouvencel :

«Il me semble à vous qui avez à besongner à cheval, vous devez estre armé d'ung harnoyx bon et seur; car une lance est moult subtille et ne treuve si petite entrée que elle ne passe ; par où elle arrive, elle est sans merci. Les plus perilleuses armes du monde sont à cheval et de la lance ; car il n'y a point de holla . $»^{11}$

10 En effet, contrairement à un coup d'épée que l'on peut contrôler et freiner à la force du poignet, un coup de lance se délivre de la pleine puissance du galop de son cheval, ce qui explique une adaptation des armes utilisées. Elles sont historiquement de deux grands types, que l'on désigne comme les lances à outrance et les lances à plaisance. Les premières ne sont ni plus ni moins que les lances de guerre, munies d'un fer affûté, que l'on trouve désigné comme un «fer émoulu » ou « fer de glaive ». Les lances à plaisance sont des lances moins dangereuses, garnies d'un fer émoussé (non affûté) ou munies d'un rochet, embout formé généralement d'une triple pointe métallique, divisant d'autant la puissance d'impact en chaque point de contact et favorisant le travail de résistance de l'armure, tout en diminuant les risques de glissement: on obtenait ainsi une lance à la fois moins dangereuse et plus facile à briser.

11 On pourrait imaginer que le rochet fut une invention ingénieuse, ayant connu une diffusion rapide et immédiate, mais il n'en est rien! En 1390, le choix est proposé entre 
les deux types de lances dans les joutes de Saint-Inglevert mais tous les jouteurs choisissent le fer de glaive ${ }^{12}$. Encore au XVI ${ }^{\mathrm{e}}$ siècle, les jouteurs se plaisent à courir des lances avec un fer affûté, sans même affronter un véritable ennemi ${ }^{13}$; le goût du risque fait partie intégrante du plaisir de la joute ${ }^{14}$.

A notre époque, une lance dure même munie d'un rochet reste considérée comme une arme très dangereuse. Aussi les lances utilisées dans les rencontres modernes se terminent-elles généralement par un embout en balsa, un bois fragile, de surcroît préfendu, qui limite voire annule le choc lors du coup. Initialement conçu pour des spectacles puis utilisé pour les rencontres compétitives, ce matériel particulier modifie la technique des jouteurs.

\section{La maîtrise technique de la joute}

Comme je l'ai souligné en introduction, la joute est une adaptation à un contexte festif de la technique d'escrime à la lance caractéristique de la chevalerie, qui est apparue au plus tard au XI ${ }^{e}$ siècle. En effet, la tapisserie de Bayeux, réalisée dans les années 1080, représente des cavaliers maniant la lance de différentes façons : certains la maintiennent à bout de bras dans une position basse, d'autres la brandissent dans une position haute, la lançant parfois comme un javelot; ce qui nous intéresse davantage pour notre propos est qu'on y trouve l'une des plus anciennes représentations connues d'une nouvelle escrime à la lance, dans laquelle l'arme est tenue en arrêt, c'est-à-dire bloquée sous l'aisselle. Dans une telle position, la puissance du coup délivré ne dépend même plus de la force du bras du cavalier mais tire pleinement profit du poids et de la vitesse du bloc homme-cheval. Le fait qu'on trouve également des représentations des autres façons de tenir la lance prouve que cette nouvelle escrime n'est pas encore uniformément adoptée à la fin du $\mathrm{XI}^{\mathrm{e}}$ siècle, mais son efficacité est telle qu'elle se généralise au XII ${ }^{e}$ siècle. Grâce à cette technique conférant une formidable puissance de pénétration, la charge à la lance devient une spécificité martiale au cœur du mode de combat de la chevalerie et participe à son statut d'élite guerrière ${ }^{15}$.

La joute permet précisément aux chevaliers de maitriser ce maniement particulier de la lance. Cette activité peut sembler au premier abord un exercice relativement simple, presque basique (une seule arme, un seul déplacement, un seul coup) or elle se révèle bien plus subtile qu'il n'y paraît. Elle requiert en effet, sur un cheval au galop qu'il faut diriger d'une seule main, de viser avec une lance mesurant environ trois mètres en avant de la main (c'est-à-dire entre la main et la pointe), et de viser de surcroît une cible elle-même mouvante, tout en étant équipé d'une armure modifiant la perception de ses mouvements et de son environnement, surtout par la limitation du champ visuel.

Une exigence équestre non négligeable dans la réussite d'un jouteur consiste à avoir un cheval allant en ligne droite, ce qui, comme nous l'avons souligné, n'est absolument pas instinctif lorsqu'il s'agit de croiser un autre cheval dont le cavalier est armé d'une lance. De nombreux exemples donnent à voir des jouteurs ratant leur cible à cause d'un écart de leur monture, tel Boucicaut, dont le cheval se dérobe avant le choc lors de sa première course au fer de glaive contre le chevalier anglais Sicart de la Barde, ce qui entraîne la colère du futur maréchal de France ${ }^{16}$.

16 Pour ce qui est de la maîtrise de l'arme, le jouteur maniant sa lance doit montrer des capacités remarquables de précision et de force. En matière de précision, la longueur de 
l'arme est un handicap certain, que l'entraînement régulier doit permettre de surmonter. Ainsi, Juan Quixada de Reayo, dans sa Doctrina des arte de la cavalleria de 1548, observe-t-il qu'un mouvement de la largeur d'un doigt à l'endroit où repose la lance devient ample de deux paumes au niveau de la pointe de la lance, autrement dit qu'un faux mouvement de quelques centimètres au niveau de la main entraîne un énorme déplacement de la pointe de la lance. Même si Quixada ne le dit pas, nous pouvons relever qu'à l'inverse, un jouteur compétent peut, d'un subtil mouvement de poignet, compenser le mouvement relativement large induit par le galop du cheval, si tant est que celui-ci adopte une allure régulière. L'auteur espagnol considère d'ailleurs qu'un chevalier compétent qui ne rencontre aucun problème avec son cheval doit toucher sa cible à chaque fois ${ }^{17}$. Cela était loin d'être le cas de la majorité, mais s'avérait véritablement possible. On relève en effet de nombreux cas de jouteurs ratant complètement leur adversaire, mais aussi de jouteurs extrêmement précis, capables de toucher à chaque passage une cible réduite ${ }^{18}$.

Une fois la lance guidée avec précision, le problème de la force, autre facteur de réussite fondamental de la joute, se pose sous deux aspects : non seulement la capacité à délivrer un coup puissant, mais aussi à l'encaisser. Pour les jouteurs médiévaux, désarçonner le chevalier adverse est un coup bien plus réussi que de mettre à terre le chevalier et son cheval, alors même que la puissance développée pour réussir un tel coup doit être impressionnante ${ }^{19}$. Et pourtant, cette considération a sa logique : si un jouteur et sa monture s'écroulent ensemble au sol, la défaillance peut alors être attribuée au cheval, le cavalier pouvant faire valoir qu'il a tenu bon dans sa selle. Le désarçonnement est donc le cas type de victoire incontestable, car il démontre à la fois la capacité à frapper du vainqueur et l'incapacité à résister du perdant ${ }^{20}$. Quant au fait de résister, il faut préciser que chaque impact est ressenti par les deux adversaires, celui qui reçoit le coup bien sûr, mais aussi celui qui le porte: au milieu du XIV ${ }^{e}$ siècle, Geoffroi de Charny, dans les Demandes pour la joute, évoque la possibilité d'être désarçonné par son propre coup ${ }^{21}$ !

Cette double exigence de force et de résistance conduit à la mise en place d'une équitation spécifique, adaptée à la nécessité absolue de se caler puissamment dans sa selle. Le roi du Portugal Dom Duarte, dans son traité sur l'équitation rédigé au début du $\mathrm{XV}^{\mathrm{e}}$ siècle, insiste à de multiples reprises sur la nécessité d'être fermement monté. Le fait de tenir sa lance bloquée en arrêt sous l'aisselle est central mais ne se suffit pas: le jouteur doit également adopter une position lui permettant de faire corps avec son cheval pour former un bloc, une sorte de projectile vivant ${ }^{22}$.

Imaginons un jouteur adoptant une position que nous qualifierons de moderne ou de classique, le dos droit, les jambes à la verticale, les genoux plus ou moins pliés; un espace apparaitrait tout naturellement entre son bassin et le troussequin de la selle. En cas d'impact de lance suffisamment puissant, le bassin reculerait dans la selle et viendrait heurter le troussequin, ce qui créerait un choc au niveau des reins. En tendant légèrement les jambes en avant (voir la flèche A sur le schéma), le cavalier cale son bassin contre le troussequin (B) et évite cette première déconvenue. En revanche, le bassin peut alors devenir un point de rotation : puisque l'ensemble du corps ne peut plus reculer, la force transmise par le coup donné (C) ou reçu (D) doit bien être encaissée d'une quelconque manière, et de nombreux jouteurs se retrouvent ainsi ployés sur la croupe de leur cheval ${ }^{23}$. En plus de tendre les jambes, le jouteur doit donc pencher en avant le haut de son corps. La puissance reçue est alors transmise au maximum vers le bas, dans le troussequin (E), se trouvant ainsi amortie par le bloc homme-cheval (F). 


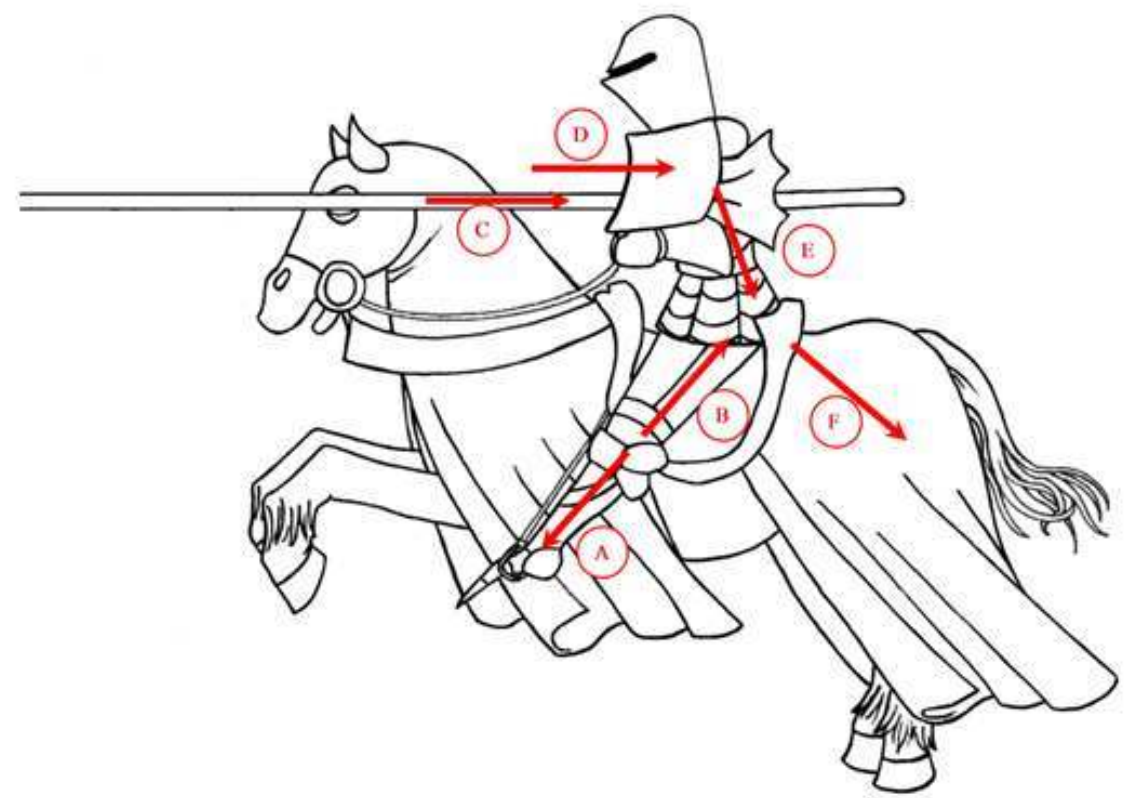

Dessin : P. Fournier.

Schéma : L. Forster

Ainsi, la position individuelle de chaque cavalier doit permettre un choc très puissant, mais ce dernier est aussi dépendant de la position relative des cavaliers l'un par rapport à l'autre. Bien entendu, plus les jouteurs passent proches l'un de l'autre, plus les coups sont portés de façon directe, c'est-à-dire qu'ils se rapprochent d'un choc frontal. A ce propos, les conséquences techniques de la barrière centrale doivent être bien évaluées. Certains historiens considèrent que cet équipement augmente l'angle d'incidence et diminue donc la puissance de frappe; ainsi, selon Claude Gaier, «la barrière oblige à aborder l'adversaire de côté et non de front $\aleph^{24}$. Il ne faudrait toutefois pas exagérer l'artificialisation induite par la barrière : lors des batailles, les chevaliers se chargent-ils parfaitement de front? Un choc véritablement frontal est impossible, ou du moins n'est certainement pas souhaitable, puisqu'il implique une collision entre les deux chevaux. Certes, la barrière facilite grandement la gestion du cheval, mais son impact ne doit pas être surestimé. Sans barrière, le souci d'éviter la collision peut amener les jouteurs à laisser une distance trop importante entre eux et à se rater complètement. En définitive, le recours à la «toile » rend l'exercice moins périlleux tout en permettant de se croiser facilement au plus près. Les joutes compétitives contemporaines, par le matériel qu'elles utilisent, ne reproduisent pas les mêmes conditions que les joutes médiévales. Les lances utilisées sont composées, en avant de la partie servant à placer la main, d'environ deux mètres de bois dur (plusieurs essences possibles dont le frêne ou le hêtre); un tube en métal fixé à l'extrémité permet de venir ficher, en la rentrant en force, la pièce d'un mètre de balsa que nous avons déjà évoquée. Ce modèle de lance a d'abord été conçu dans le milieu du spectacle, car il projette d'importants éclats de bois tout en laissant au cascadeur la possibilité de gérer son éventuelle chute : en effet, le seul bris du balsa ne crée qu'un 
impact très modéré, qui devient même presque imperceptible pour un jouteur ayant revêtu une armure complète. Cette spécificité entraîne la possibilité d'oublier le risque de choc et de sortir volontairement de la selle en avant pour briser au plus tôt sa lance sur la targe adverse, or il ne faudrait pas croire qu'une lance munie d'un embout en balsa est inoffensive, loin s'en faut. Deux différences principales constituent autant de limites à une reproduction réaliste du choc à la lance. Si un jouteur, après avoir brisé l'embout de balsa, a gardé sa lance en direction de son adversaire, un deuxième choc, cette fois-ci extrêmement puissant, est provoqué par l'impact de la partie métallique sur la cible. Premier problème : la partie restante de la lance, trop épaisse et trop courte pour se plier, est trop solide pour qu'on puisse espérer la briser ; l'intégralité de la puissance d'impact se répercute alors sur les jouteurs eux-mêmes et sur leurs chevaux. Deuxième problème : ce coup puissant est infligé alors qu'il ne reste qu'environ deux mètres de bois en avant de la main, ce qui induit un angle d'incidence supérieur à ce qu'il devrait être; cela signifie que la poussée se fait davantage sur le côté, hors de la selle, limitant l'utilité du troussequin.

En conséquence, une lance historique ne se comporterait pas comme une lance moderne, cette dernière conduisant à un choc très violent et plus latéral. Il est même légitime de se demander si cette arme moderne, qui ne peut guère se briser au-delà d'un certain seuil et absorber ainsi une partie de la puissance d'impact, peut provoquer un choc ressenti plus violemment qu'une lance historique. La recherche d'un « choc historique » serait donc un objectif important d'une approche d'archéologie expérimentale de la joute.

Toutefois, telle n'est pas la volonté des jouteurs actuels en France. Un point révélateur permettra d'en juger. L'une des principales associations voulant promouvoir la joute compétitive en France, Chevalerie Initiatique, a conçu une barrière centrale particulièrement large, qui augmente volontairement l'angle d'incidence des coups pour limiter la dangerosité des rencontres ${ }^{25}$. Le but de cet équipement et des règles qui l'accompagnent est de réduire l'échange au bris de balsa, chaque jouteur marquant ainsi des points sans donner ni subir un coup puissant. La différence technique entre joute médiévale et joute contemporaine est ici connue, acceptée et même recherchée. Cependant, une nouvelle interrogation émerge alors: si ces jouteurs s'éloignent d'un point de vue technique de la joute médiévale, sont-ils au moins proches de la mentalité d'un jouteur médiéval, voulant à tout prix sortir vainqueur d'une compétition sportive?

\section{L'état d'esprit des jouteurs}

Un souci majeur des sports contemporains réside dans l'exactitude du comptage des points et du classement des compétiteurs. En étudiant les sources dont nous disposons concernant la joute, on peut être surpris devant l'imprécision et la subjectivité qui président parfois à la désignation du vainqueur: d'après Geoffroi de Charny, dans les Demandes pour la joute, on désigne vainqueur celui qui en est jugé digne « au dit de tous ${ }^{26}$, l'appréciation de ses mérites se faisant par ses pairs, qui sont eux-mêmes des guerriers.

Même le règlement de John Tiptoft, qui propose en 1466 un système pourtant rigoureux de décompte des points, prévoit encore que le vainqueur soit choisi par les dames ${ }^{27}$. Comme l'ont désormais montré plusieurs historiens, il ne faudrait pas apporter trop de crédit à une telle affirmation : les dames récompensent celui qui leur a été désigné par les juges-diseurs ${ }^{28}$. Pour arbitrer les joutes, ces derniers rédigent des listes de touches dont nous conservons quelques exemplaires, surtout pour le XVI ${ }^{\mathrm{e}}$ siècle. Ceci dit, ce système de 
décompte extrêmement précis n'est relevé que tardivement et ne remet pas forcément en question l'existence antérieure de modes de désignation du vainqueur plus hasardeux, ou en tout cas moins rigoureux.

De plus, il n'est pas rare de voir désigner un vainqueur par équipe, le meilleur de ceux du dedans et le meilleur de ceux du dehors ${ }^{29}$, si bien que l'on ne nomme pas un vainqueur suprême. Lors des nombreux défis entre chevaliers rapportés par les chroniques, emprises d'armes et pas d'armes, de nombreux duels sont interrompus avant même qu'un combattant ne prenne véritablement le dessus sur son adversaire ${ }^{30}$.

Ces combats représentent donc des occasions de montrer sa prouesse de façon absolue, pas forcément de façon relative, chaque chevalier voulant prouver qu'il est preux, mais pas nécessairement plus preux que son adversaire, d'où les nombreuses mentions de combats dont les deux adversaires ressortent «à leur grand honneur», comme dans le roman de Jean de Bueil :

"Ces armes furent faictes et acomplies, la mercy Dieu, tellement que chascun en saillist à son honneur, tant amiz que ennemiz, par la bonne conduicte du Jouvencel. $»^{31}$

Ces façons de procéder peuvent sembler incompréhensibles pour une compétition organisée de nos jours, mais les joutes compétitives actuelles sont organisées dans un contexte très différent. Historiquement, les joutes n'opposent pas de simples sportifs mais des guerriers, dont l'évaluation des performances se fait à l'aune d'une connaissance et d'une expérience du champ de bataille.

La désignation parfois floue du vainqueur et l'existence d'un référentiel guerrier influencent donc la façon de combattre des jouteurs. Une pratique largement attestée consiste ainsi à choisir des lances particulièrement grosses, plus difficiles à casser, ce qui témoigne d'une volonté d'impressionner et de montrer sa valeur plus que de marquer des points ${ }^{32}$.

30 Un détail très intéressant mérite aussi d'être relevé dans la façon de manier la lance. D’un point de vue physique, l'étude des forces exposée dans le schéma ci-dessus pourrait présenter une faille : si le but de la joute était purement de briser sa lance pour marquer un point, le plus efficace ne serait pas de frapper en dirigeant sa lance directement sur son adversaire, mais d'essayer de la relever au moment du choc pour la plier et la faire se rompre. Même avec l'intuition que telle n'était pas la volonté des jouteurs médiévaux, il m’a longtemps été impossible d'argumenter solidement dans ce sens.

31 Je m'appuierai pour ma démonstration sur un passage qui éclaire cette question, pour peu qu'on sache l'analyser. D'après sa biographie, le grand champion bourguignon Jacques de Lalaing, lors de ses performances en joute, met tout son savoir-faire à bien " asseoir » ses coups : il apparait clair que Jacques de Lalaing frappe de façon à ce que sa lance ne se casse pas facilement afin de porter le coup le plus efficace, le plus puissant possible. Face à lui, son adversaire brise également sa lance mais ne lui assène pas un coup aussi puissant $^{33}$. Ainsi, alors même que Jacques de Lalaing a multiplié les emprises d'armes et les pas d'armes et semble être la figure la plus adaptée dans l'univers des joutes médiévales pour préfigurer les athlètes contemporains, il cherche à atteindre une prouesse martiale, guerrière, et non pas une prouesse sportive.

32 Sébastien Nadot relève que " pour la plupart des chroniqueurs, le décompte n'est pas une priorité $»^{34}$; nous pouvons ajouter qu'apparemment, il ne l'était pas non plus pour les jouteurs eux-mêmes. En définitive, les jouteurs actuels tendent à s'éloigner de 
l'historicité non seulement d'un point de vue matériel, mais aussi par les règles et l'état d'esprit.

Le risque est donc important de voir apparaitre ce que l'on pourrait appeler un sport historique décontextualisé, mêlant sans distinction, dans la pratique et dans la communication vis-à-vis du public, historicité et invention moderne. En France comme dans d'autres pays, nombre de jouteurs sont des passionnés de sport plus que d'histoire médiévale. Ainsi, l'association Chevalerie initiatique défend auprès de la Fédération française d'équitation la reconnaissance de la joute comme une discipline équestre à part entière, au même titre que le concours de saut d'obstacles. Dans ce but, la barrière spécifique que nous avons déjà mentionnée contribue au renforcement des normes de sécurité. Des tournois centrés sur des exercices d'adresse individuels sont organisés avec différents centres équestres et imposent le port de bombes modernes. Dans les joutes compétitives de façon générale, la focalisation sur l'aspect sportif conduit à d'autres anachronismes, comme la représentation des pays par leurs drapeaux actuels. Autre exemple, l'association Équispérance a organisé plusieurs tournois au programme desquels figurent des jeux d'adresse avec saut et slalom qui, s'ils requièrent une solide maitrise équestre, ne s'inspirent pas de pratiques historiques ${ }^{35}$. Enfin, l'International Jousting League propose sur son site internet des tableaux avec comptage des points sur plusieurs tournois afin de proposer des classements par année ${ }^{36}$.

L'évolution de la pratique moderne de la joute a même abouti en 2012 à un résultat pour le moins surprenant: la programmation, sur la châne américaine History, d'un show de télé-réalité portant le nom évocateur de Full Metal Jousting, qui propose de suivre l'entraînement de jouteurs qui s'éliminent ensuite au fur et à mesure des émissions, avec à la clé un prix de 100000 dollars pour le vainqueur. Ici, nul besoin pour les compétiteurs de revêtir un costume médiéval et les armures ressemblent davantage à celle du héros de comics Iron Man qu'à leurs supposés modèles d'inspiration, les armures de Gestech du XVI ${ }^{\mathrm{e}}$ siècle. Tout est fait dans la promotion pour mettre en avant le risque, les sensations fortes et l'ambiance pleine de testostérone. Le désarçonnement, largement valorisé dans le système de décompte des points, est facilité de plusieurs manières : l'utilisation de selles anglaises, donc sans troussequin, l'interdiction de tenir les rênes lors de l'impact sous peine de subir des points de pénalité, et enfin le maniement de lances faites dans un bois à la fois très souple et très résistant, qui peuvent plier de façon importante sans se rompre (ce qui aurait pour effet, comme nous l'avons expliqué précédemment, d'absorber une partie du $\operatorname{choc}^{37}$ ). L'initiateur de ce show, Shane Adams, auteur du concept de joute «full-contact », considère pour sa part qu'il revient à ce que la joute était à l'origine, à savoir un sport extrême ${ }^{38}$. En réalité, l'historicité devient ici un vague prétexte à sensations fortes et on assiste quasiment à la réinvention complète d'un sport, avec ses règles et son équipement spécifiques.

Tous ces exemples mettent en évidence le lien intime entre matériel, technique, règles et état d'esprit. Que ce soit pour une recherche de sécurité et de reconnaissance officielle, ou au contraire pour créer un show impressionnant plein de sensations fortes, la pratique de la joute est dans les deux cas dénaturée car on l'éloigne de son contexte historique, on la détourne de son but originel. 


\section{Conclusion} Olivier Renaudeau ne touche actuellement pas le monde des joutes. Il est surprenant de constater qu'au Moyen Âge, la joute a d'abord été un entraînement guerrier, qui a évolué vers le sport et le spectacle, alors qu'à notre époque, le chemin parcouru s'est fait dans le sens inverse : les premières «reconstitutions » de joutes ont été faites dans le cadre de spectacles, et on assiste actuellement à la tentative d'en faire une sorte de sport, mais un sport moderne, parfois complètement déconnecté de ses racines historiques, se développant et se construisant désormais de façon indépendante. Pratiquer la joute comme un entrainement guerrier resterait la dernière étape à parcourir pour remonter son évolution.

Dans cette perspective, le choc à la lance doit focaliser toutes les attentions. Une reproduction réaliste de cet instant crucial nécessite une recherche importante sur le matériel utilisé (armure, selle, arme,...) mais aussi sur la pratique martiale et équestre des jouteurs. Un tel travail fondé sur une démarche historique rigoureuse permettrait d'ouvrir une porte sur le monde de la guerre.

En effet, approcher la réalité de ce que vivaient les combattants n'est pas chose aisée. Nous ne pourrons jamais expérimenter une bataille véritable, et il est déjà difficile d'appréhender la réalité du duel civil à l'épée, ou plus encore la réalité du duel en armure. Dans ces conditions, la particularité de la joute d'être suffisamment sécurisée au XV siècle pour que les chevaliers n'y risquent plus guère leur vie peut représenter pour le chercheur contemporain un formidable avantage: parfois décriée par les historiens comme une activité complètement édulcorée, la joute constitue au final le seul art du combat chevaleresque que nous pourrions restituer sans concession ${ }^{39}$.

\section{NOTES}

1. Sur la popularité de la joute et sa place dans l'imaginaire médiéval, voir NADOT Sébastien, Rompez les lances! Chevaliers et tournois au Moyen Âge, Paris, Autrement, coll. « Mémoires/Culture », 2010, p. 192-195.

2. RENAUDEAU Olivier, «Du folklore médiéval à l'expérimentation archéologique, la révolution culturelle de la reconstitution du Moyen Âge en Europe ", Eidôlon, 86, 2009 ; mis en ligne en septembre 2008: http://lapril.u-bordeaux3.fr/IMG/pdf/ Olivier_RENAUDEAU_Reconstitution_en_Europe.pdf, consulté le 30 novembre 2010.

3. Je renvoie le lecteur à la communication d'Audrey TUAILLON DEMESY.

4. DUBY Georges, Guillaume le Maréchal ou le meilleur chevalier du monde, Paris, Fayard, 1984, p. 110-136. Encore faut-il bien souligner que sur ce point aussi, le monde des tournois est loin de s'opposer complètement à la guerre véritable. Dans les conflits, la pratique du butin est courante et les efforts de l'Église pour condamner les affrontements entre chrétiens ont une influence sur les comportements. Citons en exemple la bataille de Brémule en 1119, durant laquelle seuls trois

e-Phaïstos, IV-1 | 2015 
chevaliers sur les neuf-cent engagés trouvèrent la mort. CONTAMINE Philippe, La guerre au Moyen Âge, Paris, PUF, 2003, p. 414.

5. NADOT, 2010, op. cit., p. 14. Pour le renforcement de la discipline dans les formations d'infanterie.

6. Barber et Barker considèrent le $\mathrm{XV}^{\mathrm{e}}$ siècle comme l'âge d'or de la joute, quand celle-ci constitue encore un art de la guerre malgré l'importance de la mise en scène. BARBER Richard et BARKER Juliet, Les tournois, traduction française de Gérard Jean-Robert, Paris, Cie 12, 1989, p. 218.

7. Précisons que le recours à la barrière centrale ne devient pas systématique. En 1492, Bayart prévoit ainsi une joute à Aire qui doit se dérouler en champ ouvert. ANGLO Sydney, « How to win at tournaments : the technique of chivalric combat ", The Antiquaries Journal, 68, 1988, p. 255. Contamine Philippe, «Les tournois en France à la fin du Moyen Âge », dans Fleckenstein Josef (dir.), Das ritterliche Turnier im Mittelalter : Beiträge $z u$ einer vergleichenden Formen- und Verhaltensgeschichte des Ritterums, Göttingen, Vandehoeck und Ruprecht, 1985, p. 444-445.

8. BARBER et BARKER, 1989, op. cit., p. 208.

9. ANGLO, 1988, op. cit., p. 255. Geoffroi de Charny évoque les risques de blessure ou de mort pour les chevaux qui entrent en collision lors de leur course. FORSTER Loïs, Les traités de Geoffroi de Charny, mémoire de Master, Université de Franche-Comté, 2008, p. 29 et 66.

10. Notons que le savoir-faire demandé au cheval lors d'une joute est bien moins complexe que les figures de haute école dont traite Carlos Pereira dans son article. En revanche, le travail de mise en confiance dans la préparation du cheval de joute est tout aussi capital, si ce n'est plus, que pour un cheval de haute école.

11. BUEIL Jean de, Le Jouvencel, suivi du commentaire de Guillaume Tringant, éd. Favre Camille et Lecestre Léon, 2 vol., Paris, 1887 et 1889, vol. II, p. 100.

12. CONTAMINE, 1985, op. cit., p. 441.

13. Lors du troisième jour du tournoi de Westminster en 1501, organisé à l'occasion du mariage du prince Arthur et de Catherine d'Aragon, les chevaliers anglais et espagnols présents s'affrontent avec des lances affûtées. RÜHL Joachim K., « Hommes et femmes dans les tournois du Moyen Âge ", CLIO. Histoire, femmes et sociétés, 23, 2006 ; mis en ligne le 01 juin 2008 : http:// clio.revues.org/index1843.html, p. 9, consulté le 26 février 2010.

14. Le roi du Portugal Dom Duarte considère qu'on ne saurait être un bon jouteur sans prendre quelque risque et conseille par exemple de baisser sa propre targe lorsque l'on a l'impression d'avoir un avantage sur son adversaire. PRETO Antonio Franco, The Royal Book of Horsemanship, Jousting and Knightly Combat. A Translation into English of King Dom Duarte's 1438 Treatise: Livro Da Ensinança De Bem Cavalgar Toda Sela, "The Art of Riding in Every Saddle", Highland Village, Chivalry Bookshelf, 2006, p. 99.

15. FLORI Jean, La chevalerie, Paris, J.-P. Gisserot, 1998, p. 47-50 ; FLORI Jean, «Armés pour le combat!", dans Contamine Philippe (dir.), Les chevaliers, Paris, Tallandier, 2006, p. 58-59 ; GAIER Claude, «La cavalerie lourde en Europe occidentale du XIIe au XVIe siècle: un problème de mentalité ", dans GAIER Claude, Armes et combats dans l'univers médiéval, t. 1, Bruxelles, de Boeck, 1995 p. 299-303.

16. Le livre des fais du bon messire Jehan le Maingre, dit Bouciquaut, mareschal de France et gouverneur de Jennes, édition critique par Lalande Denis, Genève, 1985, p. 50.

17. ANGLO, 1988, op. cit., p. 257.

18. Il est par exemple fait mention dans le Jouvencel d'un jouteur ayant frappé à sept reprises la visière du casque de son adversaire. De façon significative, Jean de Bueil conseille d'avoir pour jouter un cheval aux doulces alleures. Jean de Bueil, 1889, op. cit., vol. II, p. 101-102. Sidney Anglo relève aussi le fossé entre le niveau des différents jouteurs. ANGLO, 1988, op. cit., p. 260.

19. Sébastien Nadot arrive à la même conclusion. NADOT, 2010, op. cit., p. 131.

20. FORSTER, 2008, op. cit., p. 34-36.

21. FORSTER, 2008, op. cit., p. 36. 
22. BUTTIN François, «La lance et l'arrêt de cuirasse », Archaeologia, 99, 1965, p. 77-205

23. De nombreuses mentions dans Le Livre des faits du bon chevalier messire Jacques de Lalaing, dans CHASTELLAIN Georges, Cuvres, éd. Kervyn de Lettenhove Joseph, VIII, Bruxelles, 1866, p. 59, $66-67, \ldots$

24. ANGLO, 1988, op. cit., p. 256 ; GAIER Claude, «Technique des combats singuliers d'après les auteurs 'bourguignons' du XV' siècle », Le Moyen Âge, 91, 1985, p. 438.

25. En ligne : http://www.chevalerieinitiatique.sitew.fr/, consulté le 2 mai 2012.

26. FORSTER, 2008, op. cit., p. 40-41.

27. NADOT, 2010, op. cit., p. 131-132.

28. RÜHL, 2006, op. cit., p. 13 ; Barber et Barker, 1989, op. cit., p. 206-207 et 217.

29. FORSTER, 2008, op. cit., p. 40-41; Contamine, 1985, op. cit., p. 425, 430-431 et 439.

30. GAIER, 1985, op. cit., p. 445-446.

31. BUEIL Jean de, 1889 , op. cit., vol. II, p. 105

32. ANGLO, 1988, op. cit., p. 256 et 260.

33. Le Livre des faits de messire Jacques de Lalaing, 1866, op. cit., p. 55 et 59.

34. NADOT, 2010, op. cit., p. 129-130.

35. En ligne : http://tournoi-de-chevalerie.over-blog.com/, consulté le 4 mai 2012.

36. En ligne : http://users.skynet.be/hackamores/IJL/IJL.htm, consulté le 4 mai 2012.

37. Une lance historique, taillée en cône, serait plus rigide et plus cassante, et ne confèrerait donc pas le même impact.

38. En ligne : http://www.history.com/shows/full-metal-jousting, consulté le 4 mai 2012.

39. Dans les mois qui ont suivi la communication dont cet article rend compte, un groupe de jouteurs s'est justement mis à présenter des joutes particulièrement respectueuses de la réalité historique. J'invite le lecteur désireux d'en savoir plus à se renseigner sur le tournoi de Sankt Wendel, le premier qui s'est tenu dans cette forme, durant l'été 2012 : http://turnier.sanktwendel.de/

\section{RÉSUMÉS}

La joute est la pratique martiale la plus codifiée de la fin du Moyen Âge. La joute est encore (de nouveau) pratiquée aujourd'hui dans le cadre du spectacle, de la reconstitution historique et même du sport. Afin de saisir l'apport et les perspectives d'une pratique expérimentale de la joute, nous nous intéresserons aux conditions dans lesquelles se pratique la joute, puis à la maîtrise technique qu'elle requiert, et enfin à l'état d'esprit dans lequel les jouteurs s'adonnent à cette activité. Pour chacune de ces parties, nous analyserons d'une part la joute médiévale et d'autre part les pratiques actuelles qui visent à la reconstituer (ou du moins à l'évoquer).

The joust is the most codified martial art of the late Middle Ages. The joust is still (again) practiced today for three reasons: offer a good show to general public, make historical research, and even practice a new sport. In order to point the contributions and perspectives of testing joust today, this paper examine the technical conditions of a joust, then the craft and technical knowledge it requires, and finally the state of mind of different jousters. For each part, we will analyze one hand medieval jousting and other current practices. 
INDEX

Mots-clés : histoire des techniques, Europe, Moyen-Âge, 21e siècle, geste technique, combat, expérimentation

Keywords : history of technology, Europa, middle ages, 20th century, technical gesture, fight, testing

\section{AUTEUR}

\section{LOIIS FORSTER}

Doctorant allocataire du Ministère de la Défense, Université Lille 3

Président de l'association Ad Honores 